\title{
Superscaling Predictions for Neutral Current Quasielastic Neutrino-Nucleus Scattering
}

\author{
M. C. Martínez, ${ }^{1}$ J. A. Caballero, ${ }^{2}$ T. W. Donnelly, ${ }^{3}$ and J. M. Udías ${ }^{1}$ \\ ${ }^{1}$ Grupo de Física Nuclear, Departamento de Física Atómica, Molecular y Nuclear, Universidad Complutense de Madrid, \\ 28040 Madrid, Spain \\ ${ }^{2}$ Departamento de Física Atómica, Molecular y Nuclear, Universidad de Sevilla, 41080 Sevilla, Spain \\ ${ }^{3}$ Center for Theoretical Physics, Laboratory for Nuclear Science and Department of Physics, Massachusetts Institute of Technology, \\ Cambridge, Massachusetts 02139, USA \\ (Received 3 August 2007; revised manuscript received 22 October 2007; published 5 February 2008)
}

\begin{abstract}
The application of superscaling ideas to predict neutral-current (NC) quasielastic (QE) neutrino cross sections is investigated. The relativistic impulse approximation (RIA) using the same relativistic mean field potential (RMF) for both initial and final nucleons - a model that reproduces the experimental $\left(e, e^{\prime}\right)$ scaling function - is used to illustrate our findings. While NC reactions are apparently not well suited for scaling analyses, to a large extent, the RIA-RMF predictions do exhibit superscaling. Independence of the scaled response on the nuclear species is very well fulfilled. The RIA-RMF NC superscaling function is in good agreement with the experimental $\left(e, e^{\prime}\right)$ one. The idea that electroweak processes can be described with a universal scaling function, provided that mild restrictions on the kinematics are assumed, is shown to be valid.
\end{abstract}

DOI: 10.1103/PhysRevLett.100.052502

PACS numbers: 25.30.Pt, 13.15.+g, 24.10.Jv

Analyses of on-going and future experimental studies of neutrino reactions and oscillations at intermediate energies [1] inevitably involve nuclear targets and require accurate control of nuclear effects. One way of taking nuclear effects into account is by directly modeling them. This approach can predict the bulk of the neutrino-nucleus response, but is not capable of yielding predictions of high enough accuracy, given the present experimental demands. A second approach that has been recently proposed takes advantage of scaling ideas. Indeed, scaling has been extensively employed to analyze inclusive QE electronnucleus scattering data $[2,3]$. The data, when appropriately organized, scale to a function that is not only relatively independent of the momentum transfer (scaling of the first kind), but also independent of the nuclear target (scaling of the second kind). The simultaneous occurrence of both kinds of scaling is known as superscaling [3]. Based on these ideas, a phenomenological superscaling approach (SuSA) [4,5] can be pursued that provides a more robust way to inter-relate the various classes of electroweak processes than most direct modeling does, as long as the kinematics chosen lie in the regions where scaling applies, i.e., QE kinematics for transferred momentum in the range from roughly $500 \mathrm{MeV} / c$ to a few $\mathrm{GeV} / c$. Within SuSA, one assumes that at similar kinematics, both electron and neutrino mediated inclusive scattering reactions share the same universal scaling function, which contains the relevant information about the initial and final state nuclear dynamics explored by the probe, thereby allowing one to provide reliable and relatively model-independent predictions for neutrino-induced processes employing the $\left(e, e^{\prime}\right)$ experimental scaling function as input [4-13].

To date, most applications of scaling ideas to neutrinonucleus cross sections involved charged current (CC) pro- cesses, whose kinematics parallel the electron scattering case. However, the interaction of neutrinos with matter is mediated not only by $W^{ \pm}$bosons, but also by the neutral $Z^{0}$ boson. NC processes are relevant for oscillation experiments - for instance, it is expected that they contribute as the third most important event type for the MiniBooNE experiment at Fermilab [1]. As in the case of CC processes, predictions based on scaling ideas, when possible, are clearly demanded. The identification of CC events is relatively simple via the outgoing charged lepton, similar to what happens in inclusive $\left(e, e^{\prime}\right)$ scattering. This means that the energy and momentum transferred at the leptonic vertex are known, and thus the scaling analysis of CC neutrino-nucleus cross sections proceeds in a way identical to the electron case. However, in the case of $\mathrm{NC}$ events, the scattered neutrino is not detected, and identification of the $\mathrm{NC}$ event is usually made when (i) no final charged lepton is found and (ii) a nucleon ejected from the nucleus is detected. Even in the case that the nucleon energy and momentum can be measured, the transferred energy and momentum at the leptonic vertex will remain unknown. The kinematics of the NC process is thus different from both electron scattering and its $\mathrm{CC}$ neutrino counterpart, rendering the derivation of scaling less obvious. Nevertheless, the translation of the scaling analysis to NC processes was recently outlined in [13]. There, it was shown that the superscaling analysis of $\mathrm{NC}$ reactions in the case of the Relativistic Fermi Gas (RFG) and scattering of $1 \mathrm{GeV}$ neutrinos from ${ }^{12} \mathrm{C}$ is feasible. Said study showed how to extend the scaling analysis to $\mathrm{NC}$ processes. The RFG $\left(e, e^{\prime}\right)$ response exhibits perfect superscaling by definition [14], but it is not in accord with the magnitude or with the shape of the experimental scaling function. It has been shown that strong final-state interactions (FSI) are 
needed to describe successfully the magnitude and shape of the superscaled data, introducing also small deviations from the extracted superscaling behavior.

In this Letter, we address two crucial questions which arise when extending SuSA analyses to NC neutrino scattering in the QE region: (i) does superscaling hold for $\mathrm{NC}$ neutrino-nucleus cross sections when strong FSI are present? If so, (ii) can the $\left(e, e^{\prime}\right)$ experimental scaling function be employed to predict NC cross sections, in spite of the intrinsic differences between the two processes? To answer these questions, being aware of how scarce NC neutrino-nucleus cross section data are, we use predictions from the Relativistic Impulse Approximation (RIA) $[7,8,15-18]$, based on strong relativistic mean field potentials for both the bound and ejected nucleons (RIA-RMF). This model, as well as its corresponding semirelativistic version [9], reproduces the shape and magnitude of the experimental scaling curve extracted from $\mathrm{QE}\left(e, e^{\prime}\right)$ data, elusive for other theoretical models. Furthermore, RIARMF predicts a universal scaling function for both electron and $\mathrm{CC}$ neutrino scattering $[7,8,10]$. Here, we verify for the first time that NC QE neutrino cross sections exhibit superscaling properties even in presence of strong FSI. Insights into the universal character of the scaling function, i.e., the existence of a unique function that simultaneously describes QE electron, CC, and NC neutrino scattering on nuclei, are also provided.

In NC QE neutrino scattering, an outgoing nucleon (mass $m_{N}$ ) having energy $E_{N}$, kinetic energy $T_{N}=E_{N}-$ $m_{N}$, and angle $\theta_{k p_{N}}$ with respect to the momentum $\mathbf{k}$ of the beam is assumed to be detected. The beam energy $\varepsilon$ is also assumed to be known. These variables determine the kinematics of the process $[13,19]$. With regards to the model we employ, the NC QE neutrino-nucleus scattering is described within the impulse approximation (IA), where the nuclear current is written as a sum of single-nucleon currents. The bound nucleon states are given as self-consistent Dirac-Hartree solutions, derived within a RMF approach using a Lagrangian containing $\sigma, \omega$, and $\rho$ mesons [20]. FSI effects are included by means of the same strong RMF potentials that describe the initial bound states. A more detailed description of the model can be found in [15$17,21]$.

As usual in scaling analyses of QE scattering, we assume the inclusive $A(\nu, N) \nu^{\prime} X$ cross section to be obtained as the integrated semi-inclusive one-nucleon (proton or neutron) knockout $A\left(\nu, \nu^{\prime} N\right) X$ cross sections. In Fig. 1, we show the strong dependence of $\mathrm{NC}$ neutrino $\mathrm{QE}$ inclusive cross sections on the beam energy (provided that $\theta_{k p_{N}}$ is fixed), and on the target selected. The results are obtained with the RIA-RMF model; however, a large amount of this variation is essentially due to the neutrino-nucleus coupling strength and the variation in the position of the quasielastic peak for the different beam energies. If superscaling holds, most of this dependence disappears when dividing these cross sec-
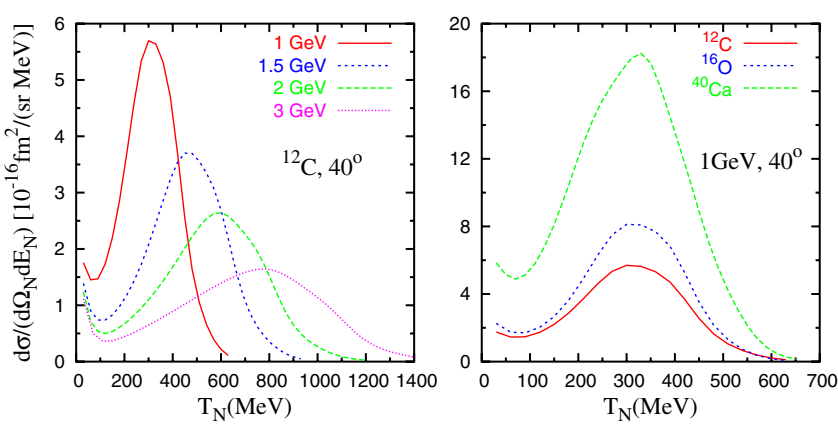

FIG. 1 (color online). NC QE differential cross section $d \sigma /\left(d E_{N} d \Omega_{N}\right)$ versus the outgoing proton kinetic energy $T_{N}$ for the reaction $(\nu, p)$. The left-hand panel corresponds to ${ }^{12} \mathrm{C}$ at different incident neutrino energies $\varepsilon$. The right-hand panel shows results at fixed $\varepsilon=1 \mathrm{GeV}$ for different target nuclei. In both panels, the outgoing nucleon detection angle is $\theta_{k p_{N}}=40^{\circ}$.

tions by the NC single-nucleon cross section given in Eq. (20) of [13] and plotting against the dimensionless scaling variable $\psi^{u}$ extracted from the RFG analysis in NC kinematics (see Eq. (26) in [13] for its explicit expression). The differences in nuclear species should also be taken into account by the superscaling analysis. Results for the so-obtained scaling function $f\left(\psi^{u}\right)$ are presented in Fig. 2.

In the left-hand panel of Fig. 2, one can see that firstkind scaling is well respected within RIA-RMF in the region of negative $\psi^{u}$-values. In other words, the large variations in the cross sections observed for different neutrino energies, are accounted for by the single-nucleon part of the cross sections, which has been factored out in obtaining the scaling function. Furthermore, the peak of the superscaling response appears approximately at the same point for all the kinematics. However, first-kind scaling is not perfect, as there is a sizeable increase in the height of the peaks of the curves, as well as a shift to $\psi^{u}>0$ for increasing beam energy. This is similar to what is observed in RIA-RMF for the inclusive $\left(e, e^{\prime}\right)$ case. Actually, the experimental $\left(e, e^{\prime}\right)$ data do leave room for some breaking of first-kind scaling in the region of positive scaling variable. First-kind scaling is very well fulfilled for
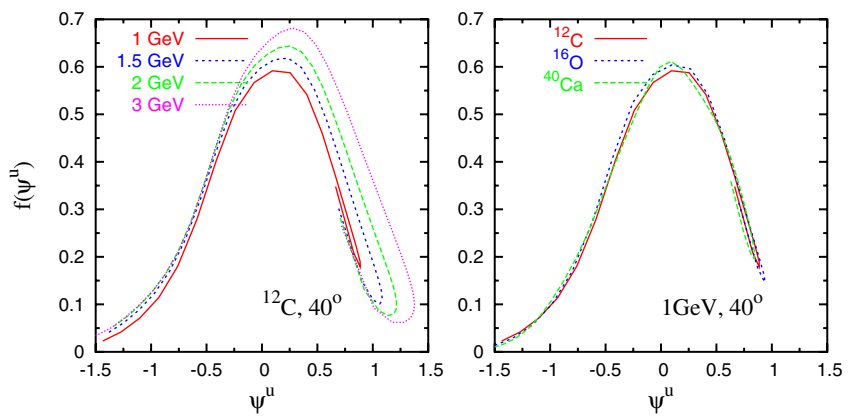

FIG. 2 (color online). NC scaling functions corresponding to the differential cross sections in Fig. 1. 
electron, $\mathrm{CC}$, and $\mathrm{NC}$ cases in the absence of FSI $[7,8,13,14,22]$. Therefore, the breakdown of scaling in Fig. 2 must be ascribed (within IA) to FSI. In the planewave limit, the dependence of the cross section on the energy of the outgoing nucleon comes mainly from kinematical effects that are taken into account in the scaling analysis. However, FSI involve a redistribution of strength that depends on the energy of the final nucleon. In other words, FSI introduce an additional, nonkinematical, dependence of the cross section on $T_{N}$. If the kinematics of the process are such that the range of energies of the ejected nucleon depends strongly on the beam energy, the nucleon will be subject to different FSI for each $\varepsilon$, and a visible breakdown of first-kind scaling will show up. This is what happens for $\theta_{k p_{N}}=40^{\circ}$, where there is a strong shift of the position of the peak of the cross section with incoming beam energy. However, for those kinematics for which the range of $T_{N}$ remains approximately the same when considering different beam energies, first-kind scaling is obtained even with FSI included, as FSI effects on the knockout nucleon are similar for different beam energies.

Incidentally, in Fig. 2, we also observe that $f\left(\psi^{u}\right)$ is, for pure kinematical reasons, a bivalued function of the scaling variable $\psi^{u}$, as the same value of $\psi^{u}$ is obtained, at fixed beam energy and nucleon angle, for two different values of the outgoing nucleon energy. In the absence of FSI (as in Ref. [13]), superscaling is a good approximation and the two values of the superscaling function for these $\psi^{u}$ are nearly equal. When FSI are present, and if the kinematics prevents superscaling, the bivalued nature of the superscaled function is revealed.

In order to understand for what kinematics good scaling of the first kind is reached even in presence of strong FSI, we look at the case of free nucleons. Figure 3 shows how $\theta_{k p_{N}}$ and $T_{N}$ are related due to energy and momentum conservation for several beam energies. For bound nucleons, neglecting Fermi motion, the cross section will be peaked at approximately the same $T_{N}$ value. From the figure, one sees that the range of $T_{N}$ spanned at fixed $\theta_{k p_{N}}$ for varying $\varepsilon$ is reduced for large angles and thus scaling of the first-kind will be much better obeyed. In general, smaller angles show larger first-kind scaling violations, while larger angles exhibit almost perfect first-kind scaling [22]. Note that this result comes through purely kinematical reasoning, and thus is model-independent to the extent that the cross section can be described within IA.

Results for scaling of the second kind are presented in the right-hand panel of Fig. 2. The superscaling functions obtained for several nuclei are almost identical, in spite of the strong difference in magnitude of the corresponding cross sections (cf. Figure 1). That is, the dependence on the nuclear species is well accounted for by the superscaling analysis. Scaling of second kind is seen to be very robust, thereby opening up a means of taking into

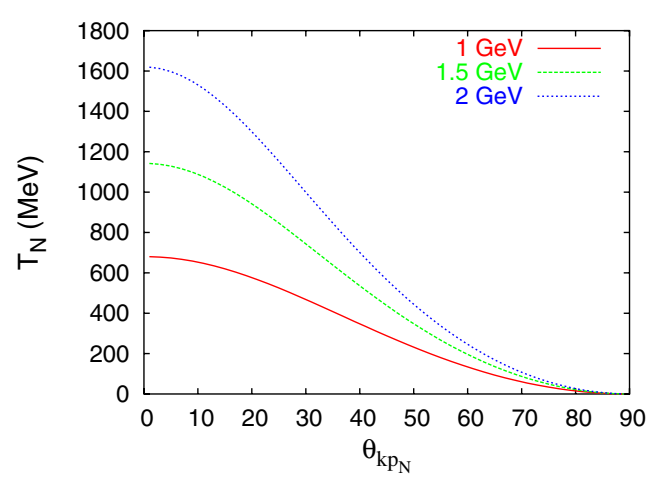

FIG. 3 (color online). Relationship between $\theta_{k p_{N}}$ and $T_{N}$ for the $\mathrm{NC}(\nu, p)$ reaction on free protons at rest. The different curves show the results for three beam energies, 1, 1.5, and $2 \mathrm{GeV}$.

account nuclear effects for different nuclei employing superscaling ideas.

The superscaling properties exhibited by NC QE neutrino-nucleus scattering suggest exploring the validity of the universal character of the scaling function for inclusive electroweak processes on nuclei, using either electrons or CC and NC neutrino probes. To the extent that this universality holds, the phenomenological SuSA approach formerly applied to predict CC neutrino-nucleus cross sections could also provide reliable, largely modelindependent predictions for $\mathrm{NC}$ processes.

In order to study whether or not this universality assumption holds also for NC processes, in Fig. 4 we compare the RIA-RMF NC superscaling function with the averaged QE experimental function obtained from the analysis of $\left(e, e^{\prime}\right)$ data, together with a phenomenological parameterization $[3,5,23]$. The RIA-RMF superscaling function has been plotted for two values of $\theta_{k p_{N}}$ for which scaling of first kind is well fulfilled $\left(60^{\circ}\right)$ or not so well $\left(40^{\circ}\right)$. Results are shown for two beam energies. As seen, the model gives rise to a $\mathrm{NC}$ scaling function that follows

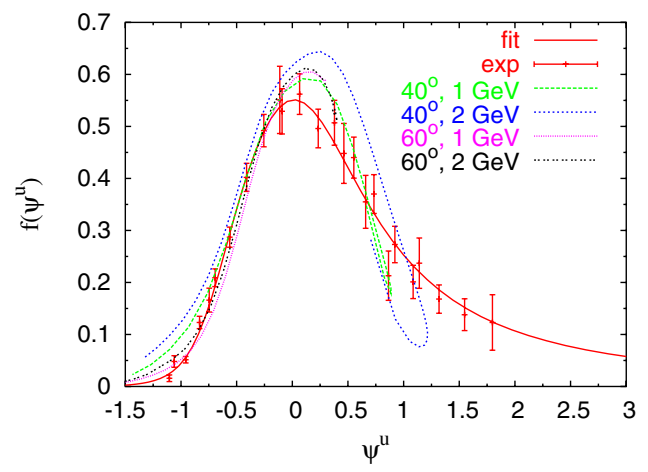

FIG. 4 (color online). NC scaling function evaluated within the RIA-RMF (dot-dashed line) approach for 40 and 60 degrees, compared with the averaged experimental function, together with a phenomenological parameterization of the data (dotted line). 
closely the behavior of the $\left(e, e^{\prime}\right)$ function, and the bivalued behavior of the superscaling function is hardly visible whenever superscaling is well respected $\left(60^{\circ}\right)$. For the case of $40^{\circ}$, breakdown of first-kind scaling is clear, the departure from the SuSA $\left(e, e^{\prime}\right)$ response visible, and the bivalued nature of the NC superscaled function is enhanced. We notice that all curves would coincide if superscaling was exactly fulfilled in both $\mathrm{NC}$ and $\left(e, e^{\prime}\right)$ cases. Since the $\left(e, e^{\prime}\right)$ and NC scaling curves are obtained under rather different kinematical situations, the scaling curves depart from each other when superscaling is not a good approximation. This supports the assumption that, under proper kinematics restrictions, a universal QE scaling function exists which is valid not only for inclusive electron and $\mathrm{CC}$ neutrino reactions, as seen in $[7,8]$, but also for $\mathrm{NC}$ processes.

In summary, we have established sufficient conditions under which a universal superscaling function could be applied both to electron and neutrino (CC or $\mathrm{NC}$ ) inclusive scattering. These conditions refer to the fact that the kinematics must be such that the range of energies spanned by the ejected nucleon is nearly independent of the incoming neutrino energy. This happens, for instance, when the angle of the ejected nucleon with regards to the beam is larger than roughly $50^{\circ}$, which happens to be the region where the cross section integrated over angles has larger values. In such a case, first-kind scaling is well respected at the $10 \%$ level even in the presence of strong FSI, and the good comparison with the experimental $\left(e, e^{\prime}\right)$ scaling function gives us confidence that SuSA can be extended to predict NC QE neutrino cross sections.

We also note that even though we have illustrated this study within the RIA-RMF model that contains strong FSI and is quite successful in reproducing the experimental electron scattering scaling function, the kinematical conditions that grant the validity of SuSA are model independent when the IA can be safely applied, that is under QE kinematics with neutrino beam energies from $\sim 500 \mathrm{MeV}$ up to a few GeV.

Work partially supported by DGI (Spain) and FEDER funds: FIS2005-01105, FPA2006-07393, FPA2006-13807, and FPA2007-62216, by the Junta de Andalucía and the INFN-CICYT, and by the Comunidad de Madrid and UCM (No. 910059 "Grupo de Física Nuclear" and No. PR1/0714895). It was also supported in part (TWD) by U.S. Department of Energy Office of Nuclear Physics under Contract No. DE-FG02-94ER40818. M. C. M. acknowledges a "Juan de la Cierva" contract from MEC. Computations were performed at the "High Performance Cluster for Physics" funded by UCM and FEDER funds.
[1] Y. Fukuda et al. (Super-Kamiokande Col.), Phys. Rev. Lett. 81, 1562 (1998); M. H. Ahn et al. (K2K), Phys. Rev. Lett. 90, 041801 (2003); E. Ables et al. (MINOS), Fermilab Report No. Fermilab-Proposal-0875, 1995; A. A. Aguilar-Arevalo et al. (MiniBooNe), Phys. Rev. Lett. 98, 231801 (2007); I. Ambats et al. (NOvA.), Fermilab Report No. Fermilab-Proposal-0929, 2004; D. Drakoulakos et al. (MINERvA), arXiv:hep-ex/0405002; S. Brice et al. (FINeSSE), arXiv:hep-ex/0402007.

[2] G. B. West, Phys. Rep. 18, 263 (1975); D. B. Day, J. S. McCarthy, T. W. Donnelly, and I. Sick, Annu. Rev. Nucl. Part. Sci. 40, 357 (1990).

[3] T. W. Donnelly and I. Sick, Phys. Rev. Lett. 82, 3212 (1999); Phys. Rev. C 60, 065502 (1999); C. Maieron, T. W. Donnelly, and I. Sick, Phys. Rev. C 65, 025502 (2002).

[4] J.E. Amaro, M. B. Barbaro, J. A. Caballero, and T. W. Donnelly, Phys. Rev. Lett. 98, 242501 (2007).

[5] J.E. Amaro et al., Phys. Rev. C 71, 015501 (2005).

[6] J.E. Amaro, M. B. Barbaro, J. A. Caballero, T. W. Donnelly, and C. Maieron, Phys. Rev. C 71, 065501 (2005).

[7] J. A. Caballero et al., Phys. Rev. Lett. 95, 252502 (2005).

[8] J. A. Caballero, Phys. Rev. C 74, 015502 (2006).

[9] J.E. Amaro, M. B. Barbaro, J. A. Caballero, T. W. Donnelly, and J. M. Udías, Phys. Rev. C 75, 034613 (2007).

[10] J. A. Caballero, J.E. Amaro, M. B. Barbaro, T.W. Donnelly, and J. M. Udías, Phys. Lett. B 653, 366 (2007).

[11] M. Martini, G. Co', M. Anguiano, and A. M. Lallena, Phys. Rev. C 75, 034604 (2007).

[12] A. N. Antonov et al., Phys. Rev. C 74, 054603 (2006); 75, 064617 (2007).

[13] J.E. Amaro, M. B. Barbaro, J.A. Caballero, and T.W. Donnelly, Phys. Rev. C 73, 035503 (2006).

[14] W. M. Alberico, A. Molinari, T.W. Donnelly, E. L. Kronenberg, and J.W. Van Orden, Phys. Rev. C 38, 1801 (1988).

[15] W. M. Alberico et al., Nucl. Phys. A 623, 471 (1997); Phys. Lett. B 438, 9 (1998); Nucl. Phys. A 651, 277 (1999).

[16] C. Maieron, M. C. Martínez, J.A. Caballero, and J. M. Udías, Phys. Rev. C 68, 048501 (2003).

[17] M. C. Martínez et al., Phys. Rev. C 73, 024607 (2006).

[18] Y. Jin, O.S. Onley, and L. E. Wright, Phys. Rev. C 45, 1333 (1992).

[19] M. B. Barbaro, A. De Pace, T. W. Donnelly, A. Molinari, and M. J. Musolf, Phys. Rev. C 54, 1954 (1996).

[20] C. J. Horowitz and B. D. Serot, Nucl. Phys. A 368, 503 (1981); Phys. Lett. B 86, 146 (1979); B. D. Serot and J. D. Walecka, Adv. Nucl. Phys. 16, 1 (1986).

[21] J. M. Udías et al., Phys. Rev. C 48, 2731 (1993); 51, 3246 (1995); 64, 024614 (2001); M. C. Martínez et al., Phys. Rev. C 69, 034604 (2004).

[22] M. C. Martínez, J. A. Caballero, T. W. Donnelly, and J. M. Udías (to be published).

[23] J. Jourdan, Nucl. Phys. A 603, 117 (1996). 